\title{
LA CRISIS SUBPRIME Y SUS EFECTOS EN LA EFICIENCIA DEL SECTOR BANCARIO CHILENO*
}

\author{
Claudio Candia Campano \\ Medardo Aguirre Gonzálezb \\ Vanessa Orellana Valenzuela ${ }^{\mathrm{c}}$ \\ Michael Gaete Morales
}

* DOI: https://doi.org/10.18601/01245996.v21n41.08 Recepción: 11-082017, modificación final: 01-04-2019, aceptación: 14-05-2019. Sugerencia de citación: Candia C., C., Aguirre G., M., Orellana V., V. y Gaete M., M. (2019). La crisis subprime y sus efectos en la eficiencia del sector bancario chileno. Revista de Economía Institucional, 21(41), 187-211.

a Investigador de la Facultad de Economía y Negocios de la Universidad de Talca, Chile, [clcandia@utalca.cl], [https://orcid.org/0000-0002-8738-2122].

b Doctor de la Universidad de Santiago de Chile y profesor de la Facultad de Economía y Negocios de la Universidad de Talca, Chile, [maguirre@ utalca.cl], [https://orcid.org/0000-0002-8683-9928].

c Investigadora de la Facultad de Economía y Negocios de la Universidad de Talca, Chile, [ceoc@utalca.cl], [https://orcid.org/0000-0003-0997-2765].

d Investigador de la Facultad de Economía y Negocios de la Universidad de Talca, Chile, [ceoc@utalca.cl], [https://orcid.org/0000-0002-0812-680X]. 


\section{La crisis subprime y sus efectos en la eficiencia del sector bancario chileno}

Resumen Este trabajo mide los efectos de la crisis subprime en la producción de eficiencia y la eficiencia técnica de la banca chilena. Se aplica el método de fronteras estocásticas de producción a un panel de datos balanceados de 14 bancos que operaban en territorio chileno entre 2004 y 2015. Los principales resultados indican que durante la crisis la producción de eficiencia y la eficiencia técnica fueron menores que en los periodos anterior y posterior, con mayores diferencias respecto del periodo posterior.

Palabras clave: eficiencia técnica, industria bancaria, fronteras estocásticas de producción; JEL: E44, O49

\section{The subprime crisis and its effects on the efficiency of the Chilean banking sector}

Abstract The objective of the research is to measure the effects of the subprime crisis on the production of efficiency and on the technical efficiency of the banking industry in Chile. For this, stochastic frontier production function methodology was used for a balanced data panel that collected information from 14 banks operating in Chilean territory for the period 2004-2015. The main results of the investigation indicate that during the crisis period, the production of efficiency and technical efficiency of the banking industry is lower, finding more important differences with respect to the period after the crisis.

Keywords: technical efficiency, banking industry, stochastic frontier production functions; JEL: E44, O49

\section{A crise do subprime e seus efeitos sobre a eficiência do setor bancário chileno}

Resumo Este documento mede os efeitos da crise do subprime sobre a eficiência da produção e sobre a eficiência técnica do sistema bancário chileno. O método das fronteiras de produção estocásticas é aplicado a um painel de dados balanceados de 14 bancos que operavam em território chileno entre 2004 e 2015 . Os principais resultados indicam que, durante a crise, a produção de eficiência e a eficiência técnica foram menores que nos períodos anterior e posterior, com maiores diferenças em relação ao período posterior.

Palavras-chaves: eficiência técnica, indústria bancária, fronteiras de produção estocásticas; JEL: E44, O49 
T a primera parte de este artículo describe la crisis subprime y luego Lel sistema bancario chileno. La segunda parte revisa los trabajos sobre la eficiencia técnica de la banca a nivel internacional, latinoamericano y nacional. Las siguientes secciones presentan el marco teórico, la metodología y los resultados. Por último se sintetizan las conclusiones.

\section{LA CRISIS SUBPRIME}

A lo largo de su historia la economía mundial ha experimentado periodos de variación en los mercados financieros internacionales, comúnmente conocidos como ciclos económicos, en los que se presentan momentos de auge, depresión y, en el peor de los casos, grandes recesiones. En 2007 la economía mundial enfrentó una fuerte crisis financiera conocida como crisis subprime, originada en Estados Unidos, marcada por la quiebra de Lehman Brothers en septiembre del 2008, y asociada a problemas de pagos de créditos hipotecarios a personas con alto nivel de riesgo. Esta crisis, el evento económico más importante desde la década de 1970, tuvo un gran impacto en los sistemas financieros (Quiggin, 2011; Moradi y Babacan, 2015).

Para entender las causas de la crisis financiera se deben revisar las modificaciones del marco regulatorio del crédito en Estados Unidos durante la década de 1980. Estas modificaciones desregularon y flexibilizaron la industria y fueron impulsadas por la crisis anterior que enfrentó el sistema financiero de ese país.

Entre los ajustes al marco anterior se destaca la aparición de un nuevo esquema hipotecario caracterizado por créditos más flexibles, nuevos instrumentos de inversión asociados a estos créditos, la disposición de las agencias gubernamentales a garantizar créditos cada vez más riesgosos y la acción negligente de las calificadoras de riesgo.

E1 esquema anterior se caracterizaba por créditos de largo plazo con tasas fijas a 30 años que permanecían en poder de las entidades emisoras hasta su vencimiento. Las modificaciones permitieron cambiar las tasas, el sistema de pago y los requisitos para solicitar crédito (Kolb, 2011). Entre los nuevos tipos de crédito hipotecario aparecieron los de tasa ajustable, los ballon mortgage y aquellos que no exigían documentos que respaldaran la capacidad de crédito de las personas. Los de tasa reajustable establecían tasas iniciales muy bajas que luego sufrían ajustes considerables que afectaban la economía familiar. Los ballon mortgage debían pagarse en su totalidad al cabo de cierto tiempo (en general a 5 años) y eran adquiridos con 
la expectativa de vender la propiedad a mayor precio antes de vencer el crédito. Los del tercer tipo (laxa información exigible) tenían tasas mucho más altas (Hubbard y Navarro, 2011).

Como parte de los instrumentos de inversión asociados a estos créditos hipotecarios más flexibles aparece el Collateralized Mortgage Obligation (CMO), un instrumento de deuda que agrupa hipotecas en un portafolio que se vende a través de títulos de participación que dan derecho al tenedor a un pago mensual proporcional a su participación en ese portafolio (Baily, Litan y Johnson, 2008).

Durante muchos años las agencias encargadas de garantizar las hipotecas preferían garantizar aquellas que cumplían el mayor número de condiciones de bajo riesgo. Entre ellas: (a) el peso de la cuota del crédito y la deuda financiera total en el ingreso mensual antes de impuestos del solicitante (no más del 28\% y el 36\% respectivamente); (b) una calificación crediticia adecuada (máximo un pago retrasado en el año anterior); (c) recursos suficientes en cuentas bancarias para cubrir la cuota inicial y el pago de las dos primeras cuotas mensuales y (d) un monto que no superara el límite establecido para créditos de su categoría (Kolb, 2011). Luego se orientaron gradualmente a garantizar créditos más riesgosos, y en el momento de la crisis tenían una alta participación en el mercado subprime (ibíd.).

Los créditos o hipotecas subprime no cumplen ninguna de las condiciones mencionadas, y los prime, de menor riesgo, satisfacen todas las condiciones. Hay dos categorías que las cumplen parcialmente, las jumbo y las Alt-A (Baily et al., 2008).

También se debe destacar la responsabilidad de las calificadoras de riesgo -como Standard y Poor's, Moody's y Fitch-en el desarrollo de la crisis. Estas agencias concedieron grado de inversión a títulos diseñados a partir de hipotecas concedidas a individuos de dudosa capacidad de pago, así incentivando la participación en la propiedad de tales títulos (Hubbard y Navarro, 2011).

Este escenario incentivó a las entidades financieras a otorgar créditos hipotecarios riesgosos sin garantías que, como parte de los nuevos instrumentos de deuda, luego se transaban (en un ambiente altas expectativas) transfiriendo el riesgo y elevando en forma artificial el valor de los títulos. Esto generó una preferencia excesiva por títulos riesgosos (hipotecas subprime) y una mayor fragilidad del sistema financiero. Cuando los acreedores no pudieron cubrir sus obligaciones se redujo el precio de las viviendas y, con ello, el de los títulos de deuda asociados a las hipotecas (Hubbard y Navarro, 2011). 
Una descripción similar de las causas de la crisis se encuentra en Corbo, Desormeaux y Schmidt (2011). Para conocer más en detalle posibles mecanismos de transmisión de las crisis financieras es recomendable revisar algunos modelos teóricos que se presentan en trabajos como los de Diamond y Dybvig (1983), Goldfajn y Valdés (1997) y Caballero y Krishnamurthy (2001). Estos trabajos identifican como principales mecanismos la contracción general del crédito, las fluctuaciones del comercio internacional, las variaciones del flujo de capitales y del precio de los bienes básicos.

Debido a las turbulencias financieras se ha hecho más importante prestar atención al comportamiento de la banca, evaluar su desempeño y conocer y medir sus niveles de eficiencia (Kristjanpoller y Saavedra, 2014), pues mayores niveles de eficiencia se traducen en aumentos del bienestar social (Mondragón, Piñeros y Pirateque, 2013).

\section{EL SISTEMA BANCARIO CHILENO}

Los sistemas financieros actúan como intermediarios que permiten reducir los costos de transacción entre el ahorro y la inversión (Fama, 1980). El sistema financiero está compuesto por el sector bancario y el no bancario. La principal actividad del sector bancario es la intermediación financiera; sus actividades secundarias son la administración de medios de pago y la actividad interbancaria. Estas se realizan principalmente a través de bancos comerciales que una vez captan dinero (captaciones) de personas y empresas (ahorradores) otorgan financiamiento (colocaciones) a otras personas y empresas (deudores). El sector no bancario está compuesto por compañías de seguros, cooperativas de ahorro y crédito, y administradoras de fondos (de inversión, de crédito, de pensión y mutuos).

E1 sistema bancario chileno está compuesto por 24 bancos comerciales. La mayoría son bancos establecidos en Chile (19), algunos son sucursales de bancos extranjeros (4) y uno es un banco estatal, como se observa en el cuadro 1, elaborado con información de la Superintendencia de Bancos e Instituciones Financieras (SBIF). Los primeros 14 bancos son los que entran en el estudio.

Las operaciones del sistema bancario chileno han crecido continuamente. La gráfica 1, elaborada con datos del Banco Central de Chile (BCCH), muestra la evolución de las colocaciones por tipo de deudor en los últimos años. Salvo en 2008-2010, esa evolución es positiva. De hecho, en 2004 las colocaciones totales llegaron a 37.232 mil millones de pesos, en 2010 aumentaron a 78.982 mil millones y 
en 2015 ascendieron a $\$ 138.385$ mil millones. Algo similar ocurre con las colocaciones como porcentaje del PIB. En 2004 representaban el 61,6\% del PIB, en 2010 el 70,8\% y en 2015 el 87,2\%, como se observa en la gráfica 2.

Cuadro 1

Participantes de la industria bancaria chilena a diciembre de 2015

\begin{tabular}{llcc}
\hline N & \multicolumn{1}{c}{ Entidad bancaria } & Tipo de banco & Tamaño \\
\hline 1 & Banco de Chile & $1^{*}$ & Grande \\
2 & Banco Internacional & $1^{*}$ & Pequeño \\
3 & Banco del Estado de Chile & $3^{*}$ & Grande \\
4 & Scotiabank Chile & $1^{*}$ & Grande \\
5 & Banco de Crédito e Inversiones & $1^{*}$ & Grande \\
6 & Corpbanca & $1^{*}$ & Grande \\
7 & Banco Bice & $1^{*}$ & Pequeño \\
8 & HSBC BANK (Chile) & $1^{*}$ & Pequeño \\
9 & Banco Santander-Chile & $1^{*}$ & Grande \\
10 & Banco Itaú Chile & $1^{*}$ & Pequeño \\
11 & The Bank of Tokyo-Mitsubishi UFJ, LTD & $2^{*}$ & Pequeño \\
12 & Banco Security & $1^{*}$ & Pequeño \\
13 & Banco Falabella & $1^{*}$ & Pequeño \\
14 & Banco Bilbao Vizcaya Argentaria, Chile (BBVA) & $1^{*}$ & Grande \\
15 & Deutsche Bank & $1^{*}$ & Pequeño \\
16 & Banco Ripley & $1^{*}$ & Pequeño \\
17 & Rabobank Chile & $1^{*}$ & Pequeño \\
18 & Banco Consorcio (ex Banco Monex) & $1^{*}$ & Pequeño \\
19 & Banco Penta & $1^{*}$ & Pequeño \\
20 & Banco Paris & $1^{*}$ & Pequeño \\
21 & Banco BTG Pactual Chile & $1^{*}$ & Pequeño \\
22 & Banco Do Brasil S.A. & $2^{*}$ & Pequeño \\
23 & JP Morgan Chase Bank, N. A. & $2^{*}$ & Pequeño \\
24 & Banco de la Nación Argentina & $2^{*}$ & Pequeño \\
\hline
\end{tabular}

1* Banco privado nacional; 2* Banco privado extranjero; 3* Banco estatal nacional.

Fuente: SBIF, elaboración propia.

\section{Gráfica 1}

Colocaciones por tipo de deudor (Miles de millones de pesos corrientes)

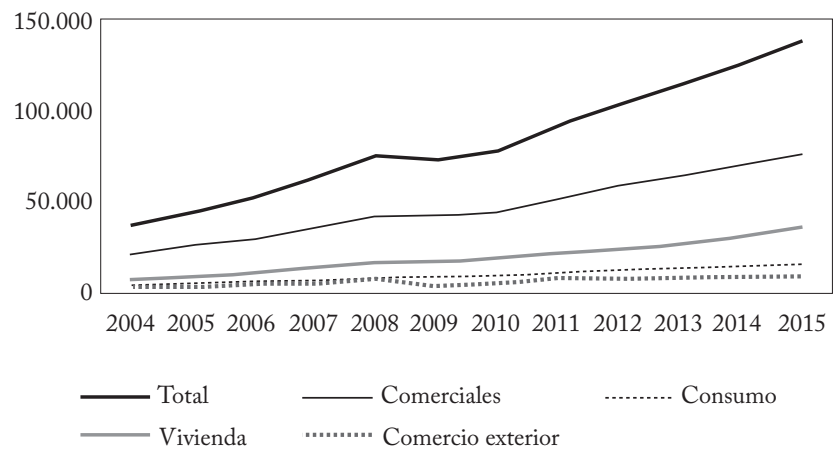

Fuente: BCCH, elaboración propia. 
La evolución de los depósitos de los ahorradores es muy parecida. Aumentaron desde 2004, excepto en el periodo de crisis (gráfica 3).

Gráfica 2

Colocaciones bancarias como porcentaje del PIB

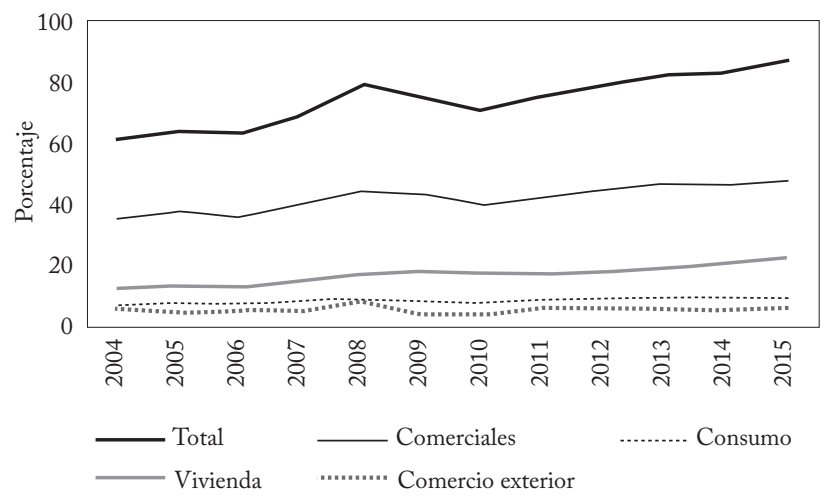

Fuente: BCCH, elaboración propia.

\section{Gráfica 3}

Depósitos de los ahorradores

(Miles de millones de pesos corrientes)

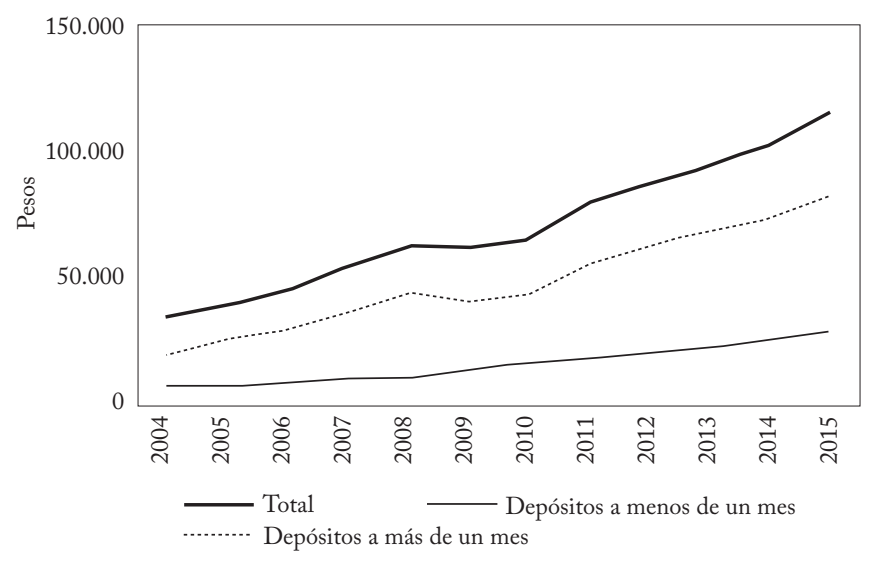

Fuente: BCCH, elaboración propia.

Por último, en la gráfica 4 se observa que los activos del sector bancario muestran una evolución similar a la de las colocaciones y los depósitos: un crecimiento continuo, solo interrumpido durante el periodo de la crisis.

Hoy, la regulación del sistema bancario chileno está a cargo de la SBIF que por mandato de la Ley General de Bancos debe supervisar 
a las empresas bancarias en salvaguardia de los depositantes u otros acreedores y del interés público, y parte de su misión es resguardar y promover la eficacia y la eficiencia en la captación y asignación de recursos.

Gráfica 4

Activos del sector bancario chileno

(Miles de millones de pesos corrientes)

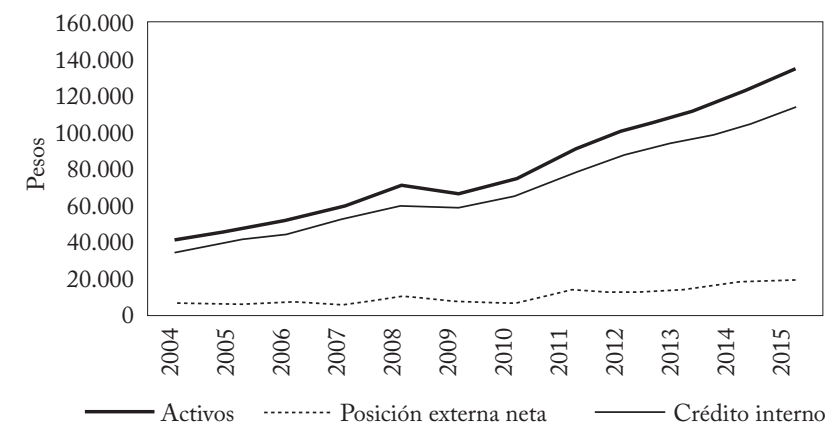

Fuente: $\mathrm{BCCH}$, elaboración propia.

\section{EFICIENCIA TÉCNICA DE LA BANCA}

Se revisaron diversos trabajos que miden la eficiencia técnica de la banca en el contexto internacional, latinoamericano y chileno. En el ámbito internacional se investigan las diferencias de eficiencia por tamaño, origen (nacional o extranjero) y propiedad (pública o privada). En los ámbitos latinoamericano y chileno la investigación no parece tener una orientación específica.

Miller y Noulas (1996) estudian la eficiencia técnica en bancos de Estados Unidos y encuentran que los más grandes y más rentables tienen mayores niveles. Kirkwood y Nahm (2006) encuentran resultados similares en la banca australiana. Isik y Hassan (2002) estiman la eficiencia técnica de los bancos turcos y concluyen que las causas de las diferencias es la heterogeneidad de las economías de escala. Y en el caso de Paquistán, Akmal y Saleem (2008) observan que los bancos públicos son menos eficientes que los extranjeros.

Parisi y Parisi (2005) miden la eficiencia técnica en bancos de todo el mundo y señalan que su carácter estatal repercute en los niveles de eficiencia. También en el ámbito internacional, Haron y Mohd (2008) encuentran que la eficiencia técnica aumentó en el periodo y que los bancos nacionales son más eficientes que los extranjeros. Otros trabajos sobre Estados Unidos también indican que los bancos nacionales son más eficientes (Chang, Hasan y Hunter, 1993; DeYoung y 
Nolle, 1996; Mahajan, Ragan y Zardkoohi, 1996). En cambio, Jemric y Vujcic (2002), que analizan la banca en Croacia encuentran que los bancos extranjeros son más eficientes que los locales.

Entre los trabajos relevantes en el ámbito latinoamericano se encuentran los de Carvallo y Kasman (2005), quienes señalan que la concentración bancaria afecta significativamente la ineficiencia. Los trabajos de Claessens, Demirgüc-Kunt y Huizinga (2001), Weill (2002) y Kasman (2005) constatan ese resultado. Staub, Da Silva y Tabak (2010) indican que los bancos extranjeros que operan en Brasil, en particular los de Europa y Estados Unidos, son más eficientes que los bancos nacionales. Badel (2002) amplía los estudios sobre la eficiencia de la banca colombiana comparándola con la de otros países latinoamericanos. Encuentra que hay homogeneidad en la eficiencia promedio aunque existe alta dispersión dentro de cada país.

En Chile se estudió la producción de la banca antes de 2002 (Skármeta, 1982 y Nauriyal, 1995) pero no su eficiencia técnica. Desde 2003, el estudio se concentra en la búsqueda de medidas de eficiencia técnica. Zúñiga y Dagnino (2003) estudian la eficiencia de la banca chilena entre 1990 y 1999, con una muestra de 24 bancos nacionales y extranjeros, y encuentran que los bancos grandes están más cerca de la frontera eficiente (paramétrica) que los pequeños y medianos. Vergara (2006), recurre a fronteras estocásticas para identificar el tipo de función más conveniente para determinar la eficiencia técnica y los costos y beneficios de la banca chilena. Concluye que es del tipo Fourier flexible y muestra que funciones menos flexibles subestiman la eficiencia. Aguirre, Bravo y Herrera (2007) comparan la eficiencia técnica de la banca chilena y alemana estimando cuatro modelos, dos no paramétricos y dos estocásticos. Su principal conclusión es que los bancos chilenos son mucho más eficientes en costos y en producción. Carreño, Loyola y Portilla (2010) estudian la eficiencia técnica de la banca chilena entre 1987 y 2007 y concluyen que ha aumentado en forma sustancial y sostenida, y que los bancos nacionales y los más pequeños son los menos eficientes.

En la revisión de la literatura se encontró que el trabajo de Wolters, Barbosa y Felício (2014) es el único que mide los efectos de la crisis subprime en la eficiencia técnica de la banca de algún país latinoamericano. Su principal resultado es que la eficiencia técnica de la banca brasileña tuvo fuerte descenso en el periodo 2007-2010. 


\section{MARCOTEÓRICO}

Esta sección describe en general la relación entre eficiencia técnica y fronteras estocásticas de producción, y luego los modelos de fronteras estocásticas tipo Battese y Coelli (1992 y 1995).

\section{FRONTERAS ESTOCÁSTICAS DE PRODUCCIÓN Y EFICIENCIA TÉCNICA}

Aunque la eficiencia técnica se define de diferentes maneras (ver, p. ej., Färe, Grosskopf y Lovell, 1985), la mayoría de la literatura la considera, para una unidad y un periodo específico, como el cociente entre la producción media (dado el nivel de factores productivos) y la producción media que la firma tendría si utilizara esos factores de la manera más eficiente posible (Battese y Coelli, 1988). Así, se puede estimar comparando la producción media efectiva con la producción media de una frontera que representa la eficiencia técnica óptima.

En la estimación se suelen utilizar métodos paramétricos y no paramétricos. En los primeros se usan técnicas econométricas y en los segundos técnicas de programación matemática para construir la frontera productiva (Tybout, 2000). Y según Din, Ghani y Mahmood (2007) la diferencia radica en el uso o no de una forma funcional específica, la cual es requerida en los métodos paramétricos ${ }^{1}$. En este trabajo se usa este último método.

Antes de llegar a una especificación generalmente aceptada de la función de la frontera había diferentes aproximaciones. Hoy se suele usar el modelo de fronteras estocásticas de producción, propuesto en forma independiente por Aigner, Lovell y Schmidt (1977) y Meeusen y Van den Broeck (1977), en el que se especifica como una función de producción con datos de corte transversal y dos componentes aleatorios, uno asociado a la presencia de ineficiencia técnica y el otro al error aleatorio. Esta especificación ha sido utilizada en numerosas aplicaciones empíricas y ha sido modificada y extendida de distintas maneras ${ }^{2}$.

Aunque es recomendable consultar los trabajos citados en la nota para conocer las modificaciones de la especificación original, se deben resaltar dos trabajos fundamentales que sintetizan, complementan y mejoran las especificaciones derivadas de la propuesta original: Bat-

1 Para mayores detalles, ver Greene (1993a) donde se hace un resumen metodológico del análisis de la eficiencia.

${ }^{2}$ Ver Førsund, Lovell y Schmidt (1980), Pitt y Lee (1981), Kalirajan (1981), Schmidt (1986), Bauer (1990), Kumbhakar, Ghosh y McGuckin (1991), Reifschneider y Stevenson (1991), Battese (1992), Greene (1993b), Huang y Liu (1994), Battese y Coelli (1988) y Battese, Coelli y Colby (1989). 
tese y Coelli (1992) y Battese y Coelli (1995). En ambos se postula la existencia de ineficiencia técnica en la producción, pero solo en el segundo se formula explícitamente un modelo complementario que explica esa ineficiencia con variables exógenas apropiadas. A continuación se revisan esas dos especificaciones.

\section{ESPECIFICACIÓN DEL MODELO BATTESE Y COELLI (1992)}

En esta se usa un procedimiento de dos etapas, primero se especifica y estima la frontera estocástica de producción y luego la eficiencia técnica. Para un panel de datos, dicha frontera se especifica como una función donde la ineficiencia técnica es una variable aleatoria distribuida de manera normal truncada que varía con el tiempo, así: $Y_{i t}=x_{i t} \beta+v_{i t}-\mu_{i t}$

$\mathrm{y}$

$\mu_{i t}=n_{i t} \mu_{i}=\exp [-n(t-T)] \mu_{i} t \in g(i) ; i=1,2, \ldots, \mathcal{N} ;$

donde $Y_{i t}$ es la producción (o su logaritmo) de $i$ en el periodo $t ; x_{i t}$ es un vector kx1 que mide la cantidad de factores productivos de $i$ en $t$; $\beta$ es un vector de parámetros desconocidos; $v_{i t}$ son errores aleatorios independientes e idénticamente distribuidos $N\left(0, \sigma_{v}^{2}\right)$, los valores $\mu_{\mathrm{i}}$ miden la ineficiencia técnica y se distribuyen de manera independiente e idéntica mediante el truncamiento no negativo de la distribución $N\left(\mu, \sigma_{\mu}^{2}\right), \eta$ es un parámetro que se debe estimar y $g(i)$ es el conjunto de periodos de tiempo $T_{i}$ entre los $T$ periodos considerados de los que se tienen observaciones para las $i$ unidades.

Para estimar la frontera estocástica de producción mediante estimadores de máxima verosimilitud se usa la reparametrización sugerida por Battese y Corra (1977), que consiste en remplazar $\sigma_{v}^{2}$ y $\sigma_{\mu}^{2}$ con $\sigma^{2}=\sigma_{v}{ }^{2}+\sigma_{\mu}^{2}$ y $\gamma=\sigma_{\mu}^{2} /\left(\sigma_{v}^{2}+\sigma_{\mu}^{2}\right)$, donde $\gamma$ debe estar entre 0 y 1 a fin de tener un buen valor de partida en el proceso iterativo.

E1 logaritmo de la función de verosimilitud (que se presenta en el apéndice de Battese y Coelli, 1992) se expresa en términos de $\beta_{i}, \sigma^{2}, \gamma$ y $\eta, y$ los estimadores de máxima verosimilitud se obtienen derivando parcialmente la función con respecto a esos parámetros. Y para estimar la eficiencia técnica, dadas las ecuaciones (1) y (2), se ha demostrado (ver el anexo de Battese y Coelli, 1992) que el estimador con menor error cuadrático medio de $i$ en $t$ es:

$$
E\left[\exp \left(-\mu_{i t}\right) \mid E_{i}\right]=\left\{\frac{1-\varphi\left[\eta_{i i} \sigma_{i}^{*}-\left(\mu_{i}^{*} / \sigma_{\sigma^{*}}^{*}\right)\right]}{1-\varphi\left(-\mu_{i}^{*} / \sigma_{i}^{*}\right)}\right\} \exp \left[-\eta_{i t} \mu_{i}^{*}+\frac{1}{2} \eta_{i i}^{2} \sigma_{i}^{*}\right]
$$


donde $E_{i}$ es el vector $\left(T_{i} 1\right)$ de los valores $E_{i t}$ asociados a los periodos de tiempo observados por $i$, donde $E_{i t}=v_{i t}-\mu_{i t}$.

$$
\begin{gathered}
\mu_{i}^{*}=\frac{\mu \sigma_{v}^{2}-\eta_{i}^{\prime} E_{i} \sigma_{\mu}^{2}}{\sigma_{v}^{2}+\eta_{i}^{\prime} \eta_{i} \sigma_{\mu}^{2}} \\
\sigma_{i}^{* 2}=\frac{\sigma_{v}^{2} \sigma_{u}^{2}}{\sigma_{v}^{2}+\eta_{i}^{\prime} \eta_{i} \sigma_{\mu}^{2}}
\end{gathered}
$$

donde $\eta_{i}$ es el vector $T_{i} x 1$ de los valores $\eta_{i \mathrm{t}}$ asociados a los periodos observados por la unidad $i$ y $\varphi$ es la función de distribución de probabilidad normal estandarizada.

\section{ESPECIFICACIÓN DEL MODELO BATTESE Y COELLI (1995)}

En esta especificación se usa un modelo para medir los efectos de la ineficiencia técnica dentro de la frontera estocástica, con datos de panel. Y también un procedimiento de dos etapas, en el que primero se especifica y estima la frontera estocástica de producción (que incorpora variables que miden los efectos de la ineficiencia) y luego se estima la eficiencia técnica. La frontera estocástica es la siguiente:

$Y_{i t}=\chi_{i t} \beta+v_{i t}-\mu_{i t}$

donde $Y_{i t}$ es la producción (o su logaritmo) de $i$ en $t ; x_{i t}$ es un vector kx1 que mide la cantidad de factores productivos de $i$ en $t, \beta$ es un vector de parámetros desconocidos; $v_{\text {it }}$ son errores aleatorios independientes e idénticamente distribuidos $\mathrm{N}\left(0, \sigma_{v}^{2}\right)$, los valores $\mu_{\mathrm{i}}$ miden la ineficiencia técnica y se distribuyen de manera independiente e idéntica mediante el truncamiento no negativo de la distribución $N\left(\mu, \sigma_{\mu}^{2}\right)$; $z_{i t}$ es un vector $1 \mathrm{xm}$ de variables exógenas asociadas a la ineficiencia técnica de producción y $\delta$ un vector $m x 1$ de coeficientes desconocidos.

La ecuación (6) especifica la frontera estocástica de producción en término de factores productivos conocidos, los efectos de ineficiencia técnica $\left(\mu_{\mathrm{it}}\right)$ son función de variables exógenas $\left(z_{i t}\right)$ y del vector de coeficientes desconocidos $\delta$.

Las variables exógenas del modelo de ineficiencia pueden incluir algunos factores productivos de la frontera estocástica y los efectos de ineficiencia técnica $\left(\mu_{\mathrm{it}}\right)$ se especifican así:

$\mu_{i t}=z_{i t} \beta+w_{i t}$

donde la variable aleatoria $w_{i t}$ se determina mediante el truncamiento de la distribución normal con media 0 y varianza $\sigma^{2}$. 
Igual que antes, para estimar simultáneamente los parámetros de la frontera estocástica de producción y los parámetros del modelo de efectos de ineficiencia técnica mediante estimadores de máxima verosimilitud se usa la reparametrización sugerida por Battese y Corra. E1 logaritmo de la función de verosimilitud y las derivadas parciales de sus parámetros se presenta en el apéndice de Battesse y Coelli (1993).

Al final, la eficiencia técnica de producción de $i$ en $t$ se define así:

$$
\exp \left(-\mu_{i t}\right)=\exp \left(-z_{i t} \delta-w_{i t}\right)
$$

\section{METODOLOGÍA}

Esta sección describe el tipo de investigación, el tipo de fuente de datos utilizados, el método de análisis, los componentes de la función objetivo a optimizar y finalmente el tratamiento realizado a los datos para obtener los respectivos paneles que permitan estimar la frontera estocástica de producción, la eficiencia técnica y los efectos de la ineficiencia técnica para la industria bancaria chilena.

\section{FUENTES DE DATOS}

Los datos utilizados provienen de la información financiera mensual de la SBIF y del BCCH con los cuales se construyeron paneles de datos de los bancos chilenos durante el periodo 2004-2015. En 2004, había 31 bancos y en 2015 solo 24; 19 de ellos bancos nacionales, 4 sucursales de bancos extranjeros y un banco estatal, como se dijo antes (cuadro 1). En el análisis solo se incluyen los primeros $14 \mathrm{de}$ ese cuadro pues son aquellos de los que se tiene información para todo el periodo.

\section{DEFINICIÓN DE LAS VARIABLES}

Para estimar el producto bancario $\left(Y_{i t}\right)$ se utiliza la suma de colocaciones, préstamos e inversiones. Las colocaciones son para comercio, vivienda y consumo. Los préstamos son deudas de otros bancos con el banco en cuestión por préstamos interbancarios de liquidez, préstamos interbancarios comerciales, préstamos comerciales, préstamos en el país, préstamos en el exterior, préstamos con letras de crédito para fines generales, entre otros. Las inversiones consideran instrumentos disponibles para la venta e instrumentos hasta su vencimiento.

Los insumos bancarios incluyen los insumos no financieros ( $I N-$ $\left.F_{i t}\right)$ y los insumos financieros $\left(I F_{i t}\right)$. Los $I N F_{i t}$ incluyen el trabajo y el capital físico. El trabajo se mide mediante el sueldo mensual 
de los trabajadores; el capital físico corresponde a los activos fijos netos asociados a propiedad, planta y equipos. Este valor neto se calcula restando al valor en libros de los activos los gastos operativos, las depreciaciones, las amortizaciones, los castigos, los impuestos y las contribuciones, según el balance mensual de cada banco. Los $I F_{i t}$ se estiman mediante las captaciones bancarias. Los montos de estas variables se pasan a pesos reales de 2009 usando el deflactor del IPC.

Para estudiar los efectos de la crisis en la industria bancaria se consideran dos variables dicotómicas $\left(D_{1}\right.$ y $\left.D_{2}\right) \cdot D_{1}$ toma el valor de 1 en los periodos previos a la crisis (primer semestre de 2004 a primer semestre de 2007), y $D_{2}$ el valor de 1 para los periodos posteriores a la crisis (segundo semestre de 2009 a segundo semestre de 2015). Así, en los modelos que se estiman, el periodo de crisis (segundo semestre de 2007- primer semestre de 2009) $D_{1}$ y $D_{2}$ toman simultáneamente el valor de 0 . Para mayores detalles ver el cuadro 2 .

Cuadro 2

Componentes de la función de producción de la banca chilena (Millones de pesos de 2009)

\begin{tabular}{llll}
\hline Símbolo & \multicolumn{1}{c}{ Nombre } & \multicolumn{1}{c}{ Forma de medición } & \multicolumn{1}{c}{ Fuente } \\
\hline$Y_{i t}$ & Producto & $\begin{array}{l}\text { Saldo de colocaciones } \\
\text { Saldo de prestamos } \\
\text { Saldo de inversiones }\end{array}$ & $\begin{array}{l}\text { SBIF y BCCH } \\
\text { SBIF y BCCH }\end{array}$ \\
\hline$I N F_{i t}$ & $\begin{array}{l}\text { Insumos no finan- } \\
\text { cieros }\end{array}$ & $\begin{array}{l}\text { Trabajo: sueldo de los trabajadores } \\
\text { Capital físico: saldo de activos fijos }\end{array}$ & $\begin{array}{l}\text { SBIF y BCCH } \\
\text { SBIF y BCCH }\end{array}$ \\
\hline$I F_{i t}$ & $\begin{array}{l}\text { Insumos finan- } \\
\text { cieros }\end{array}$ & $\begin{array}{l}\text { Captaciones-saldo de captaciones } \\
\text { SBIF }\end{array}$ & SBIF y BCCH \\
\hline$D_{1}$ & Antes de la crisis & 1 en semestres anteriores, 0 en otro caso & - \\
\hline$D_{2}$ & $\begin{array}{l}\text { Después de la } \\
\text { crisis }\end{array}$ & 1 en semestres posteriores, 0 en otro caso & - \\
\hline
\end{tabular}

Fuente: elaboración propia.

\section{TRATAMIENTO DE LOS DATOS}

Para estimar fronteras estocásticas, se debe trabajar con un panel de datos balanceado, es decir, por cada semestre del periodo de análisis se debe tener el mismo número de unidades de corte transversal. Por ello, para corregir el desbalance generado por las fusiones bancarias de 2004 a 2015, se suman los valores del producto y los insumos de los bancos correspondiente en los años previos a la fusión.

En septiembre de 2004 el Banco Security absorbió a Dresden Bank y en junio de 2005 el Banco BCI al Banco Conosur. En enero de 2008 el Banco de Chile absorbió a Citibank S.A. y en noviembre de 2009 Scotiabank al Banco del Desarrollo. 
Otra consideración importante antes de estimar las fronteras estocásticas es la imposibilidad de aplicar esta técnica en presencia de valores nulos o negativos del producto o los insumos. Por ello se eliminaron algunas unidades de corte transversal (bancos) que incumplían esta condición: el Banco Do Brasil S.A., Banco de la Nación Argentina, el Deutsche Bank y el JP Morgan Chase Bank N.S entre los extranjeros. Los tres primeros presentaban valores nulos en la cuenta de insumos no financieros, y el cuarto valores nulos en el producto bancario en el saldo de inversiones. Los bancos nacionales eliminados fueron el Banco Ripley, Rodabank Chile, Banco Consorcio, Banco París, Banco Penta y BTG Pactual Chile, pues presentaban valores nulos del producto o los insumos en algunos intervalos del periodo, que abarca 24 semestres entre 2004 y 2015.

La muestra quedó entonces conformada por los 14 primeros bancos del cuadro 1. La eliminación de los bancos mencionados solo tiene un efecto marginal en la representatividad de la muestra porque solo cubren el 2,2 de las colocaciones bancarias.

\section{MÁXIMA VEROSIMILITUD PARA ESTIMAR LA FRONTERA}

Como el error total del modelo no se distribuye en forma normal porque el componente asociado a la ineficiencia $\mu_{\mathrm{it}}$ no se distribuye así, no se puede utilizar el método de mínimos cuadrados ordinarios (MCO). Entre los métodos alternativos para estimar la función de la frontera se encuentran el método clásico con datos de panel (efectos fijos y aleatorios), el de mínimos cuadrados ordinarios modificado (MCOM) y el de máxima verosimilitud (MV).

Peña, Basch y Vergara (2003) señalan una ventaja del método de máxima verosimilitud frente al método clásico de datos de panel: las unidades más eficientes tienen más influencia en los resultados que las menos eficientes. Olson, Schmidt y Waldman (1980) hicieron una simulación de Monte Carlo y concluyeron que el estimador obtenido con MCOM es más eficiente en muestras pequeñas (menos de 200 datos) y que el estimador con MV es más eficiente en muestras grandes. Coelli (1995) también hizo una simulación de Monte Carlo y encontró que el estimador con $\mathrm{MV}$ es siempre mejor que el estimador con MCOM cuando la proporción de la varianza de la ineficiencia en el total de la varianza es alta, y recomienda usar siempre MV. Además, Polachek y Yoon (1995) confirmaron la consistencia de los estimadores de MV para modelos de fronteras estocásticas cuando se usan datos de panel. Para estimar la frontera y la eficiencia técnica se emplea el software Frontier 4.1, creado con ese propósito. 


\section{MODELOS PROPUESTOS}

Según Coelli, Rao, O’Donell y Batesse (2005) las funciones translogarítmica y Cobb-Douglas son dos de las más comunes para representar funciones de producción. Recomiendan algunos criterios para discriminar entre ellas y observan que ambas son similares en términos de linealidad de los parámetros y regularidad, aunque la translogarítmica es superior en flexibilidad y la Cobb-Douglas presenta mayor parsimonia. Debido a la translogarítmica no incorpora restricciones a la elasticidad de los factores productivos, a las elasticidades de sustitución ni al tipo de rendimientos a escala, aunque se debe estimar un mayor número de parámetros porque incluye el cuadrado de los factores y los productos cruzados, lo que puede dar lugar a problemas de multicolinealidad (ibíd., 2005).

En la literatura se suele usar más la forma translogarítmica que la Cobb-Douglas. Por ejemplo, Chávez y Fonseca (2012) analizan la eficiencia técnica y estructural de la manufactura mexicana y, con base en pruebas de hipótesis, concluyen que la forma translogarítmica representa mejor la frontera productiva del sector. Kumbhakar y Wang (2005) y Kneller y Stevens (2003) muestran que la forma Cobb-Douglas ha sido rechazada en otros estudios sobre la eficiencia.

Pese a la flexibilidad de la forma translogarítmica, su desventaja en parsimonia impide utilizarla en este trabajo pues, como indican Coelli et al. (2005), da lugar a problemas de multicolinealidad y a un ajuste inadecuado, en términos de significancia de las variables y de signo equivocado de los estimadores. De modo que se opta por la forma Cobb-Douglas, linealizada mediante logaritmos. Así los parámetros se pueden interpretar como elasticidades parciales del factor productivo con respecto al valor agregado.

Frontera estocástica de producción Batesse y Coelli (1992):

In $Y_{i t}=\beta_{0}+\sum_{j=1}^{2} \beta_{j} \operatorname{In} \chi_{j i t}+\sum_{j=1}^{2} \alpha_{j} D_{J}+v_{i t}-\mu_{i t}$

Frontera estocástica de producción Batesse y Coelli (1995):

$\operatorname{In} Y_{i t}=\beta_{0}+\sum_{j=1}^{2} \beta_{j} \operatorname{In} \chi_{j i t}+v_{i t}-\mu_{i t}$

$\mu_{i t}=\sum_{j=1}^{2} \alpha_{j} D_{j}+w_{i t}$

En ambos modelos los periodos anterior y posteriores a la crisis son variables dicotómicas. Pero en el modelo Batesse y Coelli (1992) explican el nivel de producción de eficiencia y en el modelo Batesse y Coelli (1995) explican la ineficiencia. 
Se proponen entonces dos modelos para probar la influencia del periodo de crisis en la producción de eficiencia y en la ineficiencia de la producción, donde $\mathrm{Y}_{i t}$ es el producto del banco $i$ en el semestre $t \mathrm{y}$ $X_{j i t}$ es el insumo $j$ del banco $i$ en el semestre $t, D_{j}: D_{1}$ toma el valor de 1 antes de la crisis y $D_{2}$ toma el valor de 1 después de la crisis; $\beta_{j}$ es el parámetro del insumo $j ; \alpha_{j}$ es el parámetro de la variable dicotómica $j ; v_{i t}$ es el error aleatorio para el banco $i$ en el semestre $t ; \mu_{i t}$ es el error asociado a la ineficiencia del banco $i$ en el semestre $t$ y $w_{i t}$ el error aleatorio del modelo de ineficiencia.

\section{RESULTADOS}

Esta sección muestra los principales resultados de la estimación del modelo del tipo Batesse y Coelli (1992) y del modelo del tipo Batesse y Coelli (1995). Se analiza la bondad del ajuste y la significancia individual de los parámetros que son propios a cada una de las fronteras estocásticas de producción estimadas. Finalmente se muestran resultados adicionales de interés basados en la estimación de la eficiencia técnica del modelo del tipo Batesse y Coelli (1992).

\section{RESULTADOS DEL MODELO BATESSE Y COELLI (1992)}

Con el fin probar la capacidad del modelo para pronosticar los niveles eficientes de producción de las unidades productivas (bancos) en cada semestre se hizo la prueba de razón de verosimilitud. Mientras que la primera prueba confirma la existencia de una frontera de producción eficiente, la segunda confirma la existencia de eficiencia técnica, que varía en el tiempo y entre bancos. Esto indica que el modelo es significativo para pronosticar los valores eficientes de producción (cuadro 3 ).

Cuadro 3

Prueba de razón de verosimilitud

\begin{tabular}{lccc}
\hline $\mathrm{H}_{0}$ & Estadístico de prueba & Valor del estadístico & Decisión \\
\hline$\beta_{1}=\beta_{2}=\alpha_{1}=\alpha_{2}$ & $\chi^{2}=-2$ loglikelihood $~$ & $\chi^{2}{ }_{\mathrm{c}}=-2(-108,84) \sim$ & $217,68>9,49$ \\
& $\chi^{2}(\mathrm{k}) ; \alpha$ & $\chi^{2}(4) ; 5 \%$ & Se rechaza $\mathrm{H}_{0}$ \\
\hline$\gamma=\eta=\mu=0$ & $\chi^{2}=-2\left[\log\right.$ likelihood $\left(\mathrm{H}_{0}\right)$ & $\chi^{2}=84,70 \sim \chi^{2}(3) ; 5 \%$ & $84,70>8,82$ \\
& $-\log$ likelihood $\left.\left(\mathrm{H}_{1}\right)\right]$ & & Se rechaza $\mathrm{H}_{0}$ \\
& $\sim \chi^{2}\left(\mathrm{k}_{1}-\mathrm{k}_{0}\right) ; \alpha$ & & \\
\hline
\end{tabular}

Fuente: elaboración propia.

Para evaluar la significancia de cada parámetro estimado se hacen las pruebas de hipótesis respectivas al nivel del 10\% (cuadro 4). La variable más significativa para explicar la producción son los insumos finan- 
cieros, que muestran una mayor elasticidad parcial en la producción, seguidos por los insumos no financieros. Por otra parte, al analizar las variables dicotómicas se observa que tanto en el periodo anterior a la crisis como en el periodo posterior el nivel de producción eficiente es mayor. Pero con respecto al periodo de crisis, solo el nivel del periodo posterior es significativamente mayor. Esto quiere decir que al llegar la crisis la producción de eficiencia es algo menor (cambio no significativo), pero una vez la crisis está en retirada, la producción de eficiencia aumenta visiblemente (cambio significativo).

Para evaluar la significancia de la ineficiencia técnica como causa de las desviaciones entre producción efectiva y producción eficiente, y su evolución en el tiempo, se hicieron pruebas de hipótesis de los parámetros $\gamma$ y $\eta$. Además, se hizo una prueba de hipótesis del parámetro $\mu$ para probar los supuestos sobre la función de distribución de probabilidad del componente asociado a dicha ineficiencia. Los resultados indican que las desviaciones con respecto a la frontera eficiente se deben ante todo (en un 97,8\% según la estimación de $\gamma$ ) a ineficiencia técnica y no al error aleatorio del modelo y que tal ineficiencia disminuye significativamente en el tiempo, lo que equivalente a decir que la eficiencia aumenta al pasar los años $(\eta>0)$.

Cuadro 4

Estimación del modelo Batesse y Coelli (1992)

\begin{tabular}{lccccc}
\hline$\beta$ & Coeficiente & Error estándar & Razón T & $\mathrm{H}_{0}$ & Decisión \\
\hline$\beta_{0}$ (constante) & 6,517 & 0,590 & 11,047 & $\beta_{0}=0$ & Rechaza \\
$\beta_{1}(\mathrm{INF})$ & 0,143 & 0,032 & 4,485 & $\beta_{1}=0$ & Rechaza \\
$\beta_{2}(\mathrm{IF})$ & 0,366 & 0,047 & 7,824 & $\beta_{2}=0$ & Rechaza \\
$\alpha_{1}\left(\mathrm{D}_{1}\right)$ & 0,011 & 0,054 & 0,206 & $\alpha_{1}=0$ & No se rechaza \\
$\alpha_{2}\left(\mathrm{D}_{2}\right)$ & 0,085 & 0,048 & 1,770 & $\alpha_{2}=0$ & Rechaza \\
Gamma $(\gamma)$ & 0,978 & 0,033 & 29,322 & $\gamma=0$ & Rechaza \\
Eta $(\eta)$ & 0,011 & 0,026 & 4,118 & $\eta=0$ & Rechaza \\
$\mathrm{Mu}(\mu)$ & $-4,222$ & 8,653 & $-0,488$ & $\mu=0$ & No se rechaza \\
\hline
\end{tabular}

Pruebas para un nivel de significancia del 10\%.

Fuente: elaboración propia.

\section{RESULTADOS DEL MODELO BATTESE Y COELLI (1995)}

Al estimar la frontera estocástica de producción de la banca con el modelo que mide los efectos de la ineficiencia técnica se tiene, según la primera prueba de razón de verosimilitud (cuadro 5), que es significativo para pronosticar los valores eficientes de producción. De acuerdo con la segunda prueba, los efectos de ineficiencia técnica son significativos para pronosticar los niveles de producción de eficiencia. 
Cuadro 5

Prueba de razón de verosimilitud

\begin{tabular}{lccc}
\hline $\mathrm{H}_{0}$ & Estadístico de prueba & $\begin{array}{c}\text { Valor estadístico } \\
\text { de prueba }\end{array}$ & Decisión \\
\hline$\beta_{1}=\beta_{2}=0$ & $\chi_{\mathrm{c}}^{2}=-2 \operatorname{loglikelihood~} \chi^{2}(2) ; \alpha$ & $\begin{array}{c}\chi_{\mathrm{c}}^{2}=-2(-130,64) \sim \\
\chi^{2}(2) ; 5 \%\end{array}$ & $\begin{array}{c}261,28>5,99 \mathrm{Se} \\
\text { rechaza } \mathrm{H}_{0}\end{array}$ \\
\hline$\gamma=\alpha_{1}=\alpha_{2}=0$ & $\begin{array}{c}\chi_{\mathrm{c}}^{2}=-2\left[\operatorname{loglikelihood}\left(\mathrm{H}_{0}\right)-\right. \\
\left.\text { loglikelihood }\left(\mathrm{H}_{1}\right)\right]\end{array}$ & $\begin{array}{c}\chi_{\mathrm{c}}^{2}=43,74 \sim \\
\chi^{2}(3) ; 5 \%\end{array}$ & $\begin{array}{c}43,74>7,815 \\
\text { Se rechaza } \mathrm{H}_{0}\end{array}$ \\
& $\sim \chi^{2}\left(\mathrm{k}_{1}-\mathrm{k}_{0}\right) ; \alpha$ & & \\
\hline
\end{tabular}

Fuente: elaboración propia.

Para evaluar la significancia de cada parámetro estimado se hacen las pruebas de hipótesis respectivas al nivel del 10\% (cuadro 6). La variable más significativa para explicar la producción son los insumos financieros, que muestran mayor elasticidad parcial en la producción, seguidos de los insumos no financieros. El análisis de las variables dicotómicas indica que en el periodo de crisis (con respecto a los periodos anterior y posterior) la ineficiencia técnica es mayor, pero solo hay diferencias significativas con respecto al periodo posterior. Esto quiere decir que al llegar la crisis se genera algo más de ineficiencia (cambio no significativo), pero una vez está en retirada, la ineficiencia disminuye notablemente (cambio significativo).

Cuadro 6

Estimación modelo del tipo Batesse y Coelli (1995)

\begin{tabular}{lccccc}
\hline$\beta$ & Coeficiente & Error estándar & Razón T & $\mathrm{H}_{0}$ & Decisión \\
\hline$\beta_{0}$ (constante) & 1,604 & 0,164 & 9,771 & $\beta_{0}=0$ & Rechaza \\
$\beta_{1}$ (INF) & 0,351 & 0,030 & 11,586 & $\beta_{1}=0$ & Rechaza \\
$\beta_{2}$ (IF) & 0,647 & 0,032 & 19,930 & $\beta_{2}=0$ & Rechaza \\
$\alpha_{1}\left(D_{1}\right)$ & $-0,690$ & 0,419 & $-1,647$ & $\alpha_{1}=0$ & No se rechaza \\
$\alpha_{2}\left(D_{2}\right)$ & $-1,022$ & 0,029 & $-34,660$ & $\alpha_{2}=0$ & Rechaza \\
Gamma $(\gamma)$ & 0,097 & 0,004 & 23,057 & $\gamma=0$ & Rechaza \\
\hline
\end{tabular}

Pruebas para un nivel de significancia del 10\%.

Fuente: elaboración propia.

\section{RESULTADOS ADICIONALES}

En cuanto a la evolución de la eficiencia técnica, al comienzo del periodo (primer semestre de 2004) se observa una eficiencia técnica promedio ponderada del $75,7 \%$ por producto, y del $77,0 \%$ por insumo; mientras que al final (segundo semestre de 2015) es del 79,7\% por producto y del $79,4 \%$ por insumo. La gráfica 5 muestra que aumenta durante gran parte del periodo, y también se observan algunas disminuciones, la más prolongada de las cuales ocurrió durante la crisis subprime (2007-2009). Cuando se acerca el periodo de crisis, el 
crecimiento de la eficiencia técnica se desacelera y luego es negativo hasta el primer semestre de 2009.

\section{Gráfica 5}

Eficiencia técnica de la banca chilena (Porcentaje)

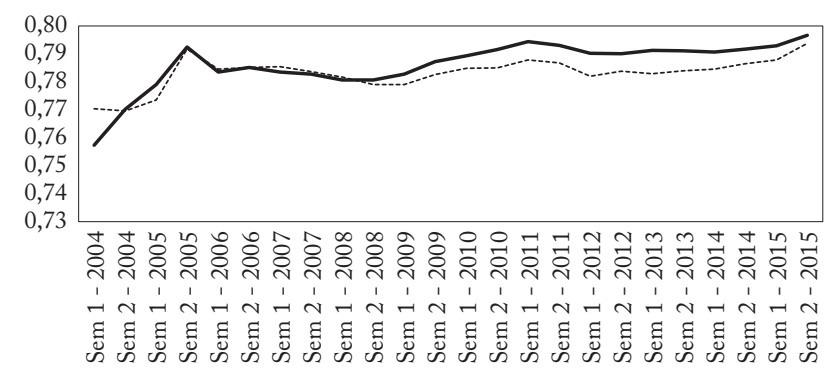

\section{- Eficiencia ponderada por producto \\ Eficiencia ponderada por insumo}

Fuente: elaboración propia.

Gráfica 6

Clasificación de los bancos chilenos por su eficiencia técnica (Porcentaje)

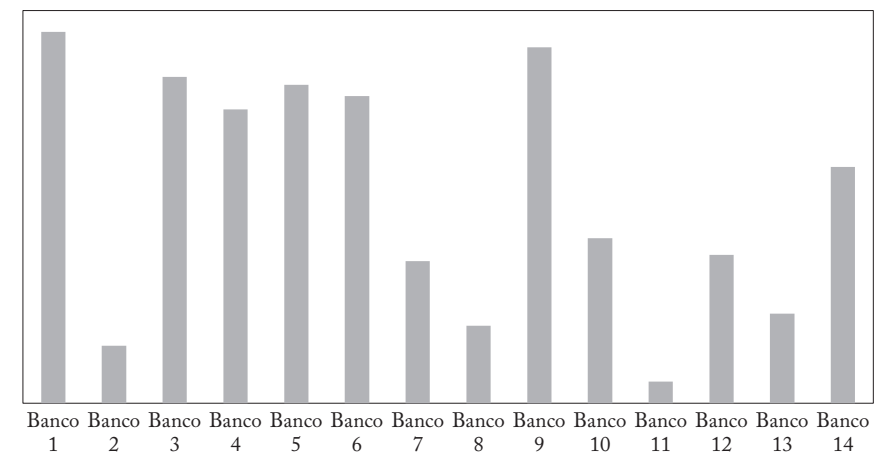

Fuente: elaboración propia.

Otro resultado importante es la clasificación de los bancos según su eficiencia técnica (gráfica 6.). Los bancos con mayor eficiencia promedio anual en el periodo analizado son el Banco de Chile (banco 1, 94,65\%), el Banco Santander-Chile (banco 9, 90,6\%), el Banco del Estado (banco 3, 83,1\%) y el Banco de Crédito e Inversiones (banco $5,81,1 \%)$. Los de menor eficiencia son el Bank of Tokyo-Mitsubishi (banco 11, 5,59\%), el Banco Internacional (banco 2, 14,7\%), el Banco HSBC (banco 8, 19,8\%) y el Banco Falabella (banco 13, 22,8\%). 
Un resultado adicional importante es el nivel de eficiencia según el tamaño de los bancos. Después de clasificarlos en grandes y pequeños según el nivel de producto y de insumos (ver el cuadro 1), la eficiencia anual promedio de los siete bancos grandes es del 80,5\% y la de los siete bancos pequeños es de apenas el 25,6\%.

\section{CONCLUSIONES}

Este artículo analiza los cambios en la producción de eficiencia y en la eficiencia técnica de la banca chilena antes y después de la crisis subprime. El primer resultado es que la crisis afectó la producción de eficiencia pues durante la crisis sus niveles fueron menores que en los periodos anterior y posterior, aunque las diferencias solo son estadísticamente significativas entre el periodo de crisis y el posterior. E1 segundo resultado es que la crisis afectó el nivel de ineficiencia técnica (y, por tanto, de eficiencia), pues en el periodo de crisis la ineficiencia técnica fue mayor ( $y$, por tanto, la eficiencia técnica menor), que en los periodos anterior y posterior, aunque las diferencias solo son estadísticamente significativas entre el periodo de crisis y el posterior. E1 tercer resultado es que entre 2004 y 2015 la eficiencia técnica general aumentó un 3,9\% cuando el factor de ponderación es el producto y un $2,4 \%$ cuando el factor es el insumo. Otro resultado importante es que los niveles de eficiencia promedio de los bancos grandes y pequeños son muy distintos: en los bancos pequeños es del $25,6 \%$ y en los grandes del $80,5 \%$.

Una vez probados los efectos de la crisis financiera sobre la eficiencia de la banca, falta investigar la relación entre dicha disminución y otras variables pertinentes. Algunos trabajos constatan el principal resultado de este trabajo en la banca de otros países, y lo interpretan de otras maneras que pueden guiar la futura investigación.

Cruz y Maudos (2016) encuentran que la crisis financiera afectó la eficiencia de la banca europea por las dificultades de los bancos para ajustar el trabajo y el capital (sucursales) en un escenario de menor demanda de crédito. Andrieș y Ursu (2016) miden el efecto de la crisis financiera en la eficiencia de la banca de la Unión Europea con métodos paramétricos y encuentran que la redujo debido al menor volumen de operaciones y a sus efectos negativos sobre la capacidad de pago de los acreedores, que forzaron a los bancos a reducir costos y a hacer provisiones por eventuales pérdidas. Singh, Elkanj, Yaacoub y Dzenopoljac (2017) examinan el impacto de la crisis en 49 de los cien principales bancos del mundo árabe -según Forbes- empleando 
métodos no paramétricos de análisis envolvente de datos. Detectan una reducción de la eficiencia durante la crisis y una recuperación en el periodo posterior. Argumentan que la pérdida de eficiencia refleja los estrechos lazos de la región con el sistema financiero internacional $y$, en particular, a los intereses que los bancos tuvieron que durante la crisis. Por último, Zeitun y Benjelloun (2012), utilizando métodos no paramétricos encuentran que la crisis financiera tuvo un efecto negativo significativo en la eficiencia de los bancos de Jordania.

\section{REFERENCIAS BIBLIOGRÁFICAS}

Aguirre, M., Bravo, G. et al. (2007). Analisis comparativo de eficiencia técnica entre la banca chilena y alemana. Revista de Matemática: Teoría y Aplicaciones, 14(2), 203-219.

Aigner, D.J., Lovell, C. A. (1977). Formulation and estimation of stochastic frontier production function models. Journal of Econometrics, 6, 21-37.

Akmal, M. y Saleem, M. (2008). Thechnical efficiency of the banking sector in pakistan. SBP Research Bulletin, 4(1), 61-80.

Andrieș, A. M. y Ursu, S. G. (2016). Financial crisis and bank efficiency: An empirical study of European banks. Economic Research-Ekonomska Istraživanja, 29(1), 485-497.

Badel, A. (2002). Sistema bancario colombiano: ¿somos eficientes a nivel internacional? DNP. Archivos de Economía 190.

Baily, M., Johnson, M. et al. (2008). The origins of the financial crisis. Washington DC: NBER paper 3.

Battese, G. E. (1992). Frontier production functions and technical efficiency: A survey of empirical applications in agricultural economics. Agricultural Economics, 7, 185-208.

Battese, G. E. y Coelli, T. J. (1988). Prediction of firm-level technical efficiencies with a generalised frontier production function and panel data. Journal of Econometrics, 38(3), 387-399.

Battese, G. E. y Coelli, T. J. (1992). Frontier production functions, technical efficiency and panel data: With application to paddy farmers in India. Journal of Productivity Analysis, 3(1-2), 153-169.

Battese, G. E. y Coelli, T. J. (1993). A stochastic frontier production function incorporating a model for technical inefficiency effects. Working Papers in Econometrics and Applied Statistics n. ${ }^{\circ} 69$. University of New England, Armidale.

Battese, G. E. y Coelli, T. J. (1995). A model for technical inefficiency effects in a stochastic frontier production function for panel data. Empirical Economics, 20, 325-332.

Battese, G. E. y Corra, G. S. (1977). Estimation of a production frontier model: With application to the pastoral zone of Eastern Australia. Australian Journal of Agricultural Economics, 21(3), 169-179.

Battese, G. E., Coelli, T. J. et al. (1989). Estimation of frontier production functions and the efficiencies of Indian farms using panel data from ICRISAT's village level studies. Journal of Quantitative Economics, 5, 327-348. 
Bauer, P. W. (1990). Recent developments in the econometric estimation of frontiers. Journal of Econometrics, 46(1-2), 39-56.

Caballero, R. y Krishnamurthy, A. (2001). International and domestic collateral constraints in a model of emerging markets crisis. Journal of Monetary Economics, 48(3), 513-548.

Carreño, J. L., Loyola, G. et al. (2010). Eficiencia bancaria en Chile: un enfoque de frontera de beneficios. Economía Chilena, 13(3), 33-65.

Carvallo, O. y Kasman, A. (2005). Cost efficiency in the Latin American and Caribbean banking systems. Journal of International Financial Markets, Institutions and Money, 15(1), 55-72.

Chang, C. E., Hasan, I. et al. (1993). Efficiency of multinational banks: An empirical investigation. Applied Financial Economics, 8(6), 689-696.

Claessens, S., Demirgüc-Kunt, A. et al. (2001). How does foreign entry affect the domestic banking market? Journal of Banking and Finance, 25(5), 891-911.

Coelli, T. J., Rao, D. S. P. et al. (2005). An introduction to efficiency and productivity analysis. $2^{\text {nd }}$ ed. Nueva York: Springer.

Coelli, T. (1995). Estimators and hypothesis test for a stochastic frontier function: a Monte Carlo analysis. Journal of Productivity Analysis, 6(4), 247-268.

Corbo, V., Desormeaux, J. et al. (2011). La gran crisis financiera de 2007-2009. Estudios Públicos, 123, 1-61.

Cruz-García, P. y Maudos, J. (2016). La situación del sector bancario español en el contexto europeo: retos pendientes. Cuadernos Económicos de ICE, 92, 81-108.

DeYoung, R. y Nolle, D. (1996). Foreign-owned banks in the US: Earning market share or buying it? Journal of Money, Credit, and Banking, 28(4), 622-636.

Diamond, D. y Dybvig, P. (1983). Bank runs, deposit insurance, and liquidity. Journal of Political Economy, 91(3), 401-419.

Din, M., Ghani, E. et al. (2007). Technical efficiency of Pakistan's manufacturing sector: A stochastic frontier and data envelopment analysis. The Pakistan Development Review, 46(1), 1-18.

Fama, E. F. (1980). Banking in a theory of financial de Eugene Fama. Journal of Monetary Economics, 6(1) 39-57.

Färe, R., Grosskopf, S. et al. (1985). The measurement of efficiency of production. Nueva York: Springer Science.

Førsund, F. R., Lovell, C. A et al. (1980). A survey of frontier production functions and of their relationship to efficiency measurement. Journal of Econometrics, 13(1), 5-25.

Goldfajn, I. y Valdés, R. (1997). Capital flows and the twin crisis: The role of liquidity. Washington DC: IMF working paper 97/98.

Greene, W. H. (1993a). The econometric approach to efficiency analysis. En H. O. Fried, C. A. et al. (eds.), The measurement of productive efficiency (pp. 68-119). Nueva York: Oxford University Press.

Greene, W. H. (1993b). Frontier production functions. Stern School of Business: NYU working paper EC 93-20. 
Haron, S. y Mohd, I. (2008). Technical efficiency of the Malaysian commercial banks: a stochastic frontier approach. Banks and Bank Systems, 3(4), 65-72.

Huang, C. J. y Liu J-T. (1994). Estimation of a non-neutral stochastic frontier production function. Journal of Productivity Analysis, 5(2), 171-180.

Hubbard, G. y Navarro, P. (2011). Seeds of destruction. Saddle River, NJ: Pearson Education.

Isik, I. y Hassan, M. (2002). Technical, scale and allocative efficiencies of Turkish banking industry. Journal of Banking y Finance, 26, 719-766.

Jemric, I. y Vujcic, B. (2002). Efficiency of banks in Croatia: A DEA approach. Comparative Economic Studies, 44(2-3), 169-193.

Kalirajan, K. (1981). An econometric analysis of yield variability in paddy production. Canadian Journal of Agricultural Economics, 29(3), 283-294.

Kasman, A. (2005). Efficiency and scale economies in transition economies: Evidence from Poland and the Czech Republic. Emerging Markets Finance and Trade, 41(2), 60-81.

Kirkwood, J. y Nahm, D. (2006). Australian banking efficiency and its relation to stock returns. The Economic Record, 82(258), 253-267.

Kneller, R. y Stevens, P. (2003). The specification of the aggregate production function in the presence of inefficiency. Economics Letters, 81(2), 223-226.

Kolb, R. (2011). The financial crisis of our time. Nueva York: Oxford University Press.

Kristjanpoller, W. y Saavedra, O. (2014). La eficiencia técnica de los bancos durante la crisis. Caso aplicado a la rentabilidad de la banca comercial en México y Chile. Contaduría y Administración, 59(1), 95-122.

Kumbhakar, S. y Wang, H. (2005). Estimation of growth convergence using a stochastic production frontier approach. Economics Letters, 88(3), 300-305.

Kumbhakar, S. C., Ghosh, S. et al. (1991). A generalized production frontier approach for estimating determinants of inefficiency in US dairy farms. Journal of Business and Economic Statistics, 9(39), 279-286.

Mahajan, A., Rangan, N. et al. (1996). Cost structures in multinational and domestic banking. Journal of Banking and Finance, 20, 238-306.

Meeusen, W. y Van den Broeck, J. (1977). Efficiency estimation from Cobb-Douglas production functions with composed error. International Economic Review, 18(2), 435-444.

Miller, S. y Noulas, A. (1996). The technical efficiency of large bank production. Journal of Banking y Finance, 20(3), 495-509.

Mondragón, L., Piñeros, J. H. et al. (2013). Eficiencia de los establecimientos bancarios (EB): una aproximación mediante modelos DEA. Banco de la República 798.

Moradi-Motlagh, A. y Babacan, A. (2015). The impact of the global financial crisis on the efficiency of Australian banks. Economic Modelling, 46(C), 397-406.

Nauriyal, B. (1995). Measures of cost economies in Chilean Banking: 1984-1991. Revista de Análisis Económico, 10(1), 71-79. 
Olson, J., Schmidt, P. et al. (1980). A Monte Carlo study of estimators of the stochastic frontier production function. Journal of Econometrics, 13(1), 67-82.

Parisi, F. y Parisi, A. (2005). Ranking de banca: 1995-2004. Departamento de Administración, Universidad de Chile.

Peña, J., Basch, M. et al. (2003). Eficiencia técnica y escala de operación en pesca pelágica: un análisis de fronteras estocásticas. Cuadernos de Economía, 40(119), 47-87.

Pitt, M. M. y Lee, L. F. (1981). Measurement and sources of technical inefficiency in the Indonesian weaving industry. Journal of Development Economics, 9(1), 43-64.

Polachek, S. W. y Yoon, B. J. (1995). Consistency of maximum likelihood estimators in stochastic frontier models. Department of Economics, State University of New York.

Quiggin, J. (2011). What have we learned from the global financial crisis? The Australian Economic Review, 44(4), 355-365.

Reifschneider, D. y Stevenson, R. (1991). Systematic departures from the frontier: A framework for the analysis of firm inefficiency. International Economic Review, 32(3), 715-723.

Schmidt, P. (1986). Frontier production functions. Econometric Reviews, 4(2), 289-328.

Singh, B., Elkanj, N. et al. (2017). Impact of financial crisis on banking sector efficiency in the Arab world. International Journal of Development and Conflict, 7(1), 49-64.

Skármeta, C. (1982). Economías de escala en la banca comercial Chilena. Cuadernos de Economía, 19(56), 69-86.

Staub, R., Da Silva, G. et al. (2010). Evolution of bank efficiency in Brazil: A DEA approach. European Journal of Operational Research, 202(1), 204-213.

Tybout, J. (2000). Manufacturing firms in developing countries: How well do they do, and Why? Journal of Economic Literature, 38(1), 11-44.

Vergara, M. (2006). Nota técnica para estimar fronteras estocásticas: una aplicación a la banca chilena. Estudios de Administración, 13(2), 47-66.

Weill, L. (2002). Banking efficiency in transition economies: The role of foreign ownership. Economics of Transition, 11(3), 569-592.

Wolters, M. E., Barbosa Do Couto, E. et al. (2014). The effects of the global financial crisis on Brazilian banking efficiency. Innovar, 24(53), 23-40.

Zeitun, R. y Benjelloun, H. (2012). The efficiency of banks and financial crisis in a developing economy: The case of Jordan. International Review of Accounting, Banking and Finance, 4(2), 28-60.

Zúñiga, S. y Dagnino, E. (2003). Medición de la eficiencia bancaria en Chile a través de fronteras estocásticas (1990-1999). ABANTE, 6(2), 83-116. 